\title{
Uso de dexmedetomidina como estrategia de sedación en resección endoscópica de tumor endotraqueal
}

\author{
Use of dexmedetomidine as sedation strategy for \\ endoscopic resection of endotracheal tumor
}

\author{
INGRID MARTínez RamíreZ, MD(1); Edgar SÁNCHeZ, MD(2); Alfredo SAAVedRa, MD(3); \\ Plutarco García HeRreros, MD ${ }^{(4)}$
}

\section{Resumen}

Hombre de 43 años, con cáncer de tiroides tipo papilar con invasión endotraqueal por masa a nivel del tercio superior de la tráquea, que requirió resección endoscópica mediante electrocirugía. Como estrategia de sedación se utilizó dexmedetomidina a fin de lograr cooperación del paciente sin provocar efectos adversos como taquicardia, hipotensión/hipertensión, o desaturación durante el procedimiento. La dexmedetomidina posee un perfil farmacológico y de seguridad que la convierte en una opción terapéutica adecuada de sedación para uso en broncoscopia intervencionista.

Palabras clave: broncoscopia, sedación consciente, dexmedetomidina.

\begin{abstract}
We report the case of a 43 year old male with papillary thyroid cancer with tracheal invasion by mass at the level of the upper third of the trachea that required endoscopic resection by electro surgery. As a sedation option we used dexmedetomidine. The patient didn't have adverse effects such as tachycardia, hypotension / hypertension, or desaturation during the procedure. Dexmedetomidine has a pharmacological and safety profile that makes it an appropriate therapeutic option for use in interventional bronchoscopy.
\end{abstract}

Keywords: bronchoscopy, conscious sedation, dexmedetomidine.

\section{Introducción}

Cada vez se realizan con mayor frecuencia procedimientos endoscópicos intervencionistas con fines curativos y paliativos, hecho que obliga al uso de opciones seguras de sedación y/o anestesia general para reducir las complicaciones y lograr la cooperación del paciente con el objetivo de facilitar el procedimiento. El empleo de opioides y benzodiacepinas se asocia con efectos adversos de orden cardiovascular y depresión respiratoria, lo cual limita su utilidad. La dexmedetomidina posee efectos simpaticolíticos, además de permitir sedación, amnesia y analgesia sin afectar la capacidad de ventilación espontánea del paciente. En la actualidad hay un debate creciente acerca de si la sedación debe ser suministrada por el broncoscopista o el anestesiólogo y a la vez un aumento en la evidencia que reporta que la sedación no administrada por anestesiólogos es segura y costoefectiva (1). Por lo anterior, la dexmedetomidina se convierte en una opción adecuada para procedimientos intervencionistas que puede ser usada por el broncoscopista de forma segura.
${ }^{(1)}$ Médico Internista. Fellow de Neumología. Universidad Nacional de Colombia.

${ }^{(2)}$ Médico Internista. Neumólogo. Profesor asistente. Unidad de Neumología, Instituto Nacional de Cancerología. Bogotá. Universidad Nacional de Colombia.

${ }^{(3)}$ Médico Internista. Neumólogo. Profesor asociado. Unidad de Neumología Instituto Nacional de Cancerología. Bogotá. Universidad Nacional de Colombia.

${ }^{(4)}$ Médico Internista. Neumólogo. Bogotá. Unidad de Neumología, Instituto Nacional de Cancerología.

Correspondencia: Ingrid Martínez Ramírez. Correo electrónico: ijmartinezr@unal.edu.co. Recibido: .27-09-2013 Aceptado: 22-12-2013. 


\section{Caso clínico}

Paciente de género masculino, de 57 años de edad, con masa de crecimiento progresivo en región anterior del cuello, quien consultó por disnea, y cuya tomografía evidenció lesión primaria de la glándula tiroides con infiltración de la pared posterolateral de la tráquea e invasión subglótica de la luz con estenosis del 70\% (figura 1). Mediante broncoscopia se confirmaron los hallazgos y requirió resección endoscópica (figura 2). Se utilizó dexmedetomidina,

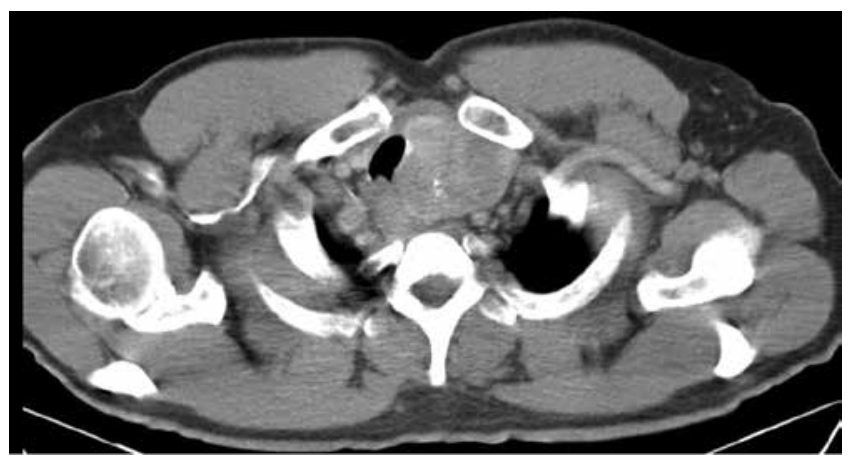

Figura 1. Visión endoscópica de lesión endoluminal en la pared posterior de la tráquea proximal.

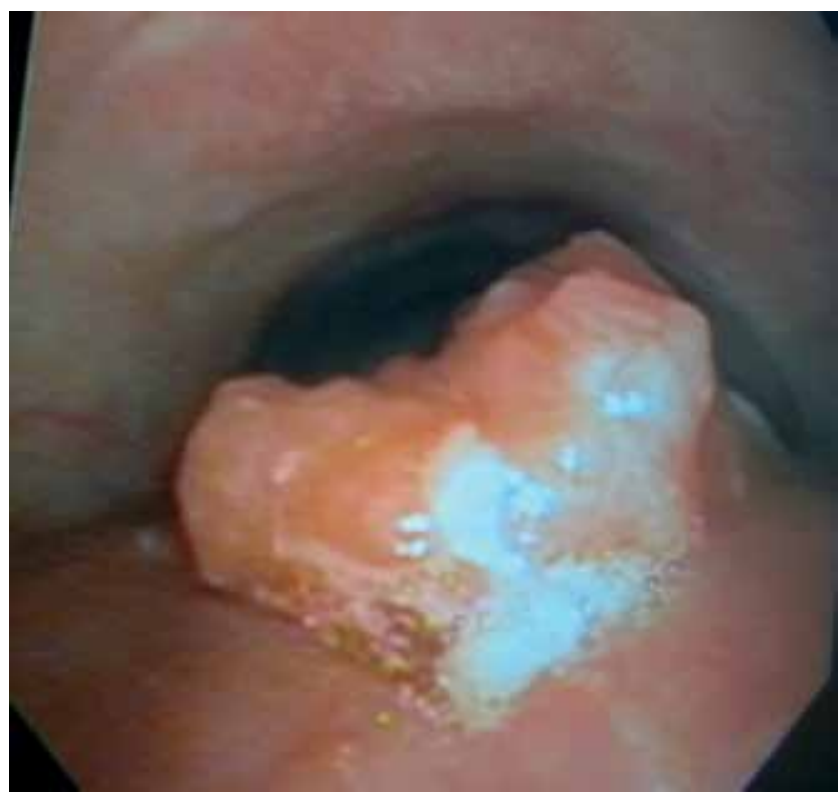

Figura 2. Cortes coronales de tomografía con lesión primaria de glándula tiroides con infiltración de la pared posterolateral de la tráquea e invasión subglótica de la luz con estenosis del $70 \%$.

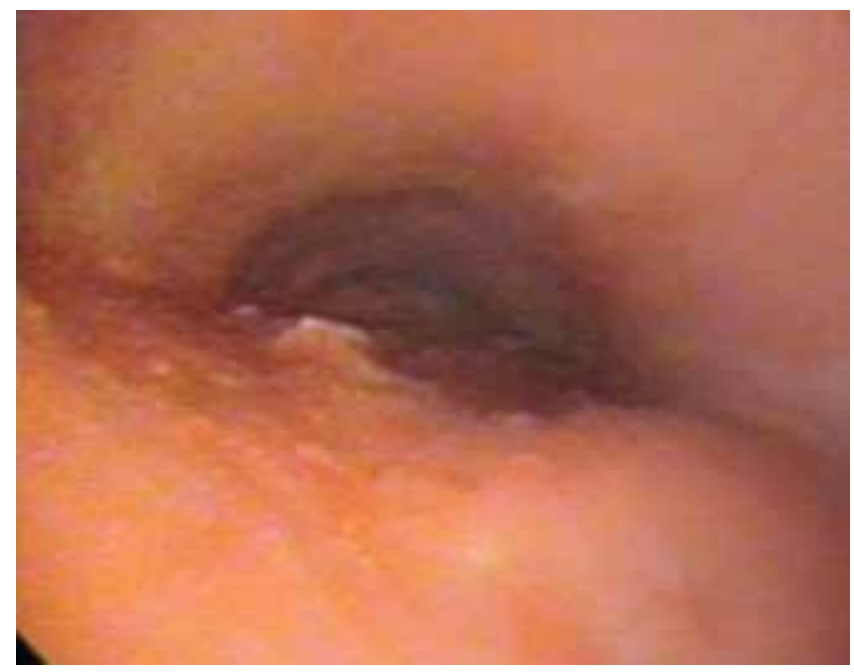

Figura 3. Visión endoscópica de la tráquea posterior al finalizar resección por electrocirugía.

bolo inicial de $10 \mathrm{mcg} / \mathrm{kg}$ en $10 \mathrm{~min}$ e infusión constante a $0,5 \mathrm{mcg} / \mathrm{kg} / \mathrm{h}$.

Necesitó, además, 120 min para completar la resección; se mantuvo puntaje de Ramsay nivel 2, con dosis total de 150 mcg de dexmedetomidina y 84 mcg de lidocaína. La frecuencia cardiaca mínima fue de $52 \mathrm{lpm}$ y la máxima de 97 . No se presentó hipoxemia, hipotensión, hipertensión, taquicardia, necesidad de intubación o suspensión del procedimiento. En una escala de 1 a 10 el paciente calificó la intensidad de la tos como 3 y manifestó que de ser necesario se repetiría el procedimiento. Se logró permeabilizar el 50\% de la luz traqueal con mejoría parcial de la sintomatología (figura 3). El reporte de biopsias confirmó carcinoma papilar de patrón célula alta, que conllevó a faringolaringectomía, tiroidectomía total y vaciamiento radical modificado por servicio de cabeza y cuello.

\section{Discusión}

Con la evolución de la broncoscopia y la posibilidad de realizar procedimientos más complejos y de duración prolongada, adquiere gran importancia el uso de sedación y el concepto de satisfacción y seguridad del paciente con el cuidado médico (1). Más del $50 \%$ de los pacientes expresan miedo a presentar cualquier tipo de disconfort con el procedimiento y 
hasta el 60\% encuentran intolerable la broncoscopia (2). Las guías actuales para broncoscopia recomiendan ofrecer sedación a los pacientes para lograr cooperación y reducir complicaciones, y anestesia general para broncoscopia rígida y procedimientos pediátricos; sin embargo, no siempre se dispone de anestesiólogo en los servicios de broncoscopia y cada vez aumenta la evidencia a favor de la seguridad del uso de sedación administrada por el broncoscopista (1).

La sedación ideal no está definida. Se proponen distintos fármacos, como midazolam o propofol, y combinaciones de benzodiacepinas y algunos opiáceos; no obstante, las propiedades farmacocinéticas que incluyen aclaramiento metabólico variable, interacciones con otros medicamentos, depresión respiratoria dependiente de dosis, sedación prolongada y alteraciones cognitivas, limitan su aplicación en el contexto de broncoscopia intervencionista (4).

La dexmedetomidina surge como una opción farmacológica en sedación para broncoscopia tanto diagnóstica como intervencionista. Se introdujo hace más de dos décadas como sedación en el ámbito del cuidado intensivo para pacientes con intubación traqueal, y rápidamente se adaptó para procedimientos que no requieren intubación traqueal. Tiene un perfil favorable de sedación, simpatolisis, analgesia y bajo riesgo de apnea. Es un agonista del adrenorreceptor alfa 2 que induce sedación por su acción en receptores del locus ceruleus, analgesia por medio de los receptores en médula espinal y atenuación de la respuesta de estrés sin depresión respiratoria significativa. Se han realizado pocos estudios en el contexto de la fibrobroncoscopia con fines diagnósticos y terapéuticos. Un estudio llevado a cabo por Ryu y colaboradores, comparó el uso de propofolremifentanil vs. propofol-dexmedetomidina, y halló una disminución significativa de la incidencia de desaturación de oxígeno en el grupo que recibió manejo con propofol y dexmedetomidina $(p=0,01)$, además de reducción en la necesidad de succión de la cavidad oral, sin diferencias significativas en el nivel de sedación, variables hemodinámicas y escalas para valoración de tos o satisfacción del paciente (5). Otro estudio comparó dexmedetomidina $v s$. midazolam durante broncoscopia flexible, y encontró que la primera mantuvo mejor saturación de oxígeno y fue igualmente bien tolerada (6).

En el caso reportado el paciente cursó con estabilidad hemodinámica, ausencia de dolor o disnea y disminución de la sensación de tos, con lo que pudo confirmarse que la dexmedetomidina ofrece efectos cardioprotectores durante los procedimientos y disminuye la respuesta al estrés (1), condiciones que la convierten en una opción farmacológica adecuada para la sedación durante la broncoscopia intervencionista.

\section{Agradecimientos y financiación}

Para el desarrollo de la presentación del caso no se contó con ningún tipo de fuente de financiación.

\section{Bibliografía}

1. José R, Shaefi S, Navani N. Sedation for flexible bronchoscopy: current and emerging evidence. Eur Respir Rev. 2013; 22 (128): 106-16.

2. Pickles J, Jeffrey M, Datta A, Jeffrey AA. Is preparation for bronchoscopy optimal? Eur Respir J. 2003; 22: 203-6.

3. Sarkiss M. Anesthesia for bronchoscopy and interventional pulmonology: from moderate sedation to jet ventilation. Curr Opin Pulm Med. 2011; 17 (4): 274-278.

4. Floyd MD, Gervasini G, Masica AL, et al. Genotype-phenotype associations for common CYP3A4 and CYP3A5 variants in the basal and induced metabolism of midazolam in European- and African-American men and women. Pharmacogenetics. 2003; 13: 595-606.

5. Ryu jh, Lee SW, Lee JH, Lee EH, Do SH, Kim CS. Randomized double-blind study of remifentanil and dexmedetomidine for flexible bronchoscopy. British J Anaesthesia. 2012; 108 (3): 503-11.

6. Liao W, Ma G, Su QG, Fang Y, Gu BC, Zou XM. Dexmedetomidine versus midazolam for conscious sedation in postoperative patients undergoing flexible bronchoscopy: a randomized study. J Int Med Research. 2012; 40: 1371-1380. 